\title{
ON THE NUMBER OF SHORTEST PATHS BY NEIGHBORHOOD SEQUENCES ON THE SQUARE GRID
}

\author{
BENEDEK NAGY \\ This paper is dedicated to Professor Béla Vizvári on his 70th birthday. \\ Received 12 December, 2018
}

\begin{abstract}
In this paper we are addressing a counting problem of discrete mathematics, more precisely of digital geometry. In the Euclidean plane the shortest path between any two points is given by the straight line segment connecting the points. In discrete mathematics, the shortest path is usually not unique, e.g., in graphs there could be several shortest paths between two vertices. In this paper, a special infinite graph, the square grid, (i.e., the usual digital plane) is used. In digital geometry there are various digital, i.e., path based distance functions. A neighborhood sequence $B$ gives the condition for each step of a $B$-path separately what type of neighborhood is used in that step. Therefore, the length and also the number of the shortest paths between two points depend not only on the respective positions (coordinate differences) of the points but also on the neighborhood sequence $B$. We give an algorithm and also closed formulae to compute the number of shortest $B$-paths.
\end{abstract}

2010 Mathematics Subject Classification: 68R05; 05C30; 52C45; 68U10

Keywords: enumerative combinatorics, digital geometry, neighborhood sequences, octagonal distances, digital distances, shortest paths, path counting, B-distances

\section{INTRODUCTION}

When we are using a computer/digital camera/tablet a digital space, i.e., the square grid with points/pixels addressed by integer coordinate pairs is taken into consideration. Working on digital spaces, e.g., on a computer screen or on digital images, the digital, path-based distances have several advantages. The digital geometry is essentially different from the Euclidean geometry. In Euclidean space there are infinitely many points between any two distinct points. In digital geometry, the space is discrete; the regular grids can be seen as special graphs. Path-based distances are based on the concept of neighborhood relation among the points of the grid. On the square grid containing points with integer coordinates there are two basic neighborhood relations: two points are considered as 1-neighbors if one of their coordinates coincides and the difference on the other is exactly 1 . A step from a point to one of its 1 -neighbor points, a 1 -step, may change one coordinate by exactly 1 . One may 
also allow diagonal movements: two points are 2-neighbors if their coordinate differences are at most 1; consequently, in a 2-step both or any of the coordinates can be changed by at most 1 . The first two digital distance functions (see [16]), from the end of 1960's, the cityblock (or Manhattan) and the chessboard distances, using these neighborhood relations in the paths, are very rough approximations of the Euclidean distance. Therefore, there are various other digital distances considered to lower the rotational dependency. Already Rosenfeld and Pfaltz, in [16], recommended to use the two types of neighborhood alternately in a path. New developments appeared approximately 20 years later. One of these approaches is the usage of neighborhood sequences giving the flexibility to change to change neighborhoods in a step of a path depending on a predefined sequence. (See $[4,5,18,19]$, where periodic neighborhood sequences were investigated; around 2000, this periodic condition was dropped to allow general, not necessarily periodic, neighborhood sequences.) A greedy algorithm that provides a shortest path between any two points by any neighborhood sequence $B$ can be found in [10], while a general formula to compute $B$-distances can be found in [12]. We should note here that digital distances, and especially $B$-distances, can be applied in various image processing tasks $[7,17]$ and also in communication networks [13], their extension for computing distances of sequences was also investigated $[14,15]$. In digital geometry, similarly as in graph theory, there is usually more than one minimal (i.e., shortest) path between two points, and it is an interesting combinatorial problem to find their numbers. Already in [16] combinatorial problems based on digital distances were investigated, namely 'path counting'. Then, a matrix algorithm is provided in [2] to count the number of shortest paths between any pair of pixels of a shape in a binary image. We should note that the number of shortest paths are also important in connection to application of networks. Further, in [3], Das gave recursive formulae for the number of shortest paths between any two points of the square grid using special distance functions, e.g., the cityblock and the chessboard distances.

In this paper, we address a more general problem: we are counting the number of shortest $B$-paths from the point $p$ to the point $q$ of the square grid, where apart from the coordinates of the points, the neighborhood sequence $B$ is also a parameter.

\section{BASIC NOTIONS AND DEFINITIONS}

Let $\mathbb{N}, \mathbb{N}^{+}$and $\mathbb{Z}$ denote the sets of nonnegative integers, positive integers and integers, respectively. Consequently, the points of the square grid are addressed by $\mathbb{Z}^{2}$. In digital geometry, and generally, in grids the concept neighborhood plays an essential role.

Let $p, q \in \mathbb{Z}^{2}$ be two points of the grid with $p=\left(x_{p}, y_{p}\right)$ and $q=\left(x_{q}, y_{q}\right)$. We say that $p$ and $q$ are 1-neighbors (cityblock neighbors) if $\left|x_{p}-x_{q}\right|+\left|y_{p}-y_{q}\right|=1$ and $p \neq q$ are 2-neighbors (chessboard neighbors) if $\left|x_{p}-x_{q}\right| \leq 1$ and $\left|y_{p}-y_{q}\right| \leq 1$. 
Notice that all 1-neighbors are also 2-neighbors, by definition. A movement from a point to one of its $i$-neighbors is called an $i$-step $(i \in\{1,2\})$.

Formally, a neighborhood sequence $B=(b(i))_{i=1}^{\infty}$ is an infinite sequence containing only the elements of the set $\{1,2\}$, i.e., $b(i) \in\{1,2\}$ for all $i \in \mathbb{N}^{+}$. A $B$-path, i.e., a path defined by $B$, from a point $p$ to a point $q$ of the grid is a sequence of points $p_{0}, p_{1}, \ldots, p_{n}$ such that $p_{0}=p$ and $p_{n}=q$, moreover $p_{i-1}$ and $p_{i}$ are $b(i)$-neighbors for every $1 \leq i \leq n$. In this way, the neighborhood sequence $B$ guides the path, in a sense, allowing or denying the diagonal steps for each step. The length of such a path is $n$, that is the number of steps in the path. Obviously, there are several $B$ paths between any two points and some of them (at least one) have a minimal length; this minimal length gives the $B$-distance $d_{B}(p, q)$ of the points $p$ and $q$. One can see that the classical cityblock and chessboard distances can be obtained by using 'constant' neighborhood sequences $B_{1}=\left(b_{1}(i)\right)_{i=1}^{\infty}$ with $b_{1}(i)=1$ for every $i \in \mathbb{N}^{+}$ and $B_{2}=\left(b_{2}(i)\right)_{i=1}^{\infty}$ with $b_{2}(i)=2$ for every $i \in \mathbb{N}^{+}$, respectively. We note here, that by the original definition of neighborhood sequences, see, e.g., [4, 5] only periodic neighborhood sequences were used, and thus, their description was done by only one period, i.e., with a finite length. The general approach used here does not restrict the study to periodic neighborhood sequences, and we use their general description by infinite sequences.

In $[9,10]$ a greedy algorithm was shown to obtain a shortest $B$-path between the input points and it was also proven to be correct. Moreover, when $B$ is fixed, the $B$-paths are translation invariant: for any point pair $p, q \in \mathbb{Z}^{2}$, any $B$-path from $p$ to $q$ has a bijective image as a $B$-path from the origin $(0,0)$ to the point $r=(p-q)=$ $\left(x_{p}-x_{q}, y_{p}-y_{q}\right)$. Consequently, shortest paths are mapped to shortest paths. The $B$-distances are symmetric, but, depending on $B$, they may not fulfill the triangular inequality. In $[10,11]$ it is proven that $B$ defines a metric if and only if the number of 2 's in its prefix sequence with length $n$ is not more than the number of 2's anywhere in the sequence among $n$ consecutive elements for all $n \in \mathbb{N}^{+}$. A formula to compute the $B$-distance is provided in $[11,12]$, now we recall it:

Let $p, q$ be points in $\mathbb{Z}^{2}$ and $B$ be a neighborhood sequence. Let $j$ and $i$ denote their coordinate differences: $j=\max \left\{\left|p_{x}-q_{x}\right|,\left|p_{y}-q_{y}\right|\right\}$ and $i=\min \left\{\left|p_{x}-q_{x}\right|,\left|p_{y}-q_{y}\right|\right\}$. Then the $B$-distance of the points is

$$
d_{B}(p, q)=\max \left\{j, k \mid j+i>\sum_{m=1}^{k-1} b(m)\right\} .
$$

\subsection{Squaring the circles}

Digital disks (and circles) are defined based on digital distances analogously to the definition of Euclidean disks (and circles).

It is well known that the disks based on cityblock distances has a 'diamond' shape (actually, it is a square standing on one of its corner) with 'stair' sides (an example is shown in Figure 1, a). We just briefly mention here that by using the digital distance 


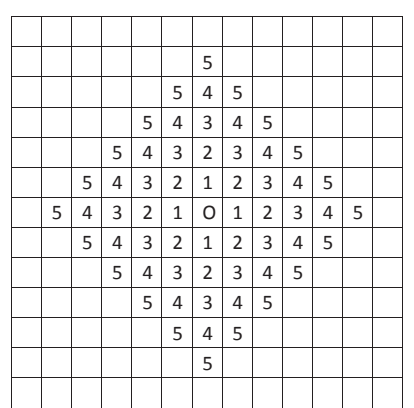

(a)

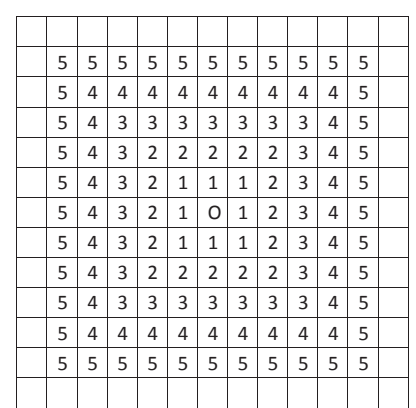

(b)

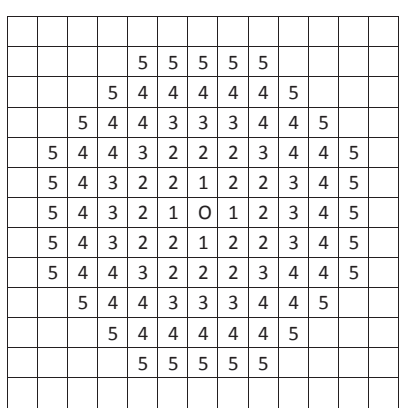

(c)

FIGURE 1. Digital disks with radius 5 based on (a) cityblock distance, (b) chessboard distance and (c) neighborhood sequence $B=$ $(1,2,1,2,1, \ldots)$ where the elements $b(i)$ with $i \geq 6$ does not play any role in the shown disks, and therefore, their values are arbitrary. The numbers typed into the pixels shows their distance from the center (marked by 'O'). (One may also observe the disks having radius $r$ less than 5 by considering those pixels that have value not more than $r$.)

based on chessboard neighbors, the digital disks are squares (with straight line sides, see Figure 1, b).

In general case, the digital disk based on a neighborhood sequence is an octagon. The previous very rough, square estimations of Euclidean circles become 'more round' (e.g., by measuring it with the isoperimetric ratio). Roughly saying, the number of the used 1's gives the length of the 'stair'-sides, while the horizontal and vertical (straight line) sides have the length double more than the used 2's (see, e.g., Figure 1, c). For this reason, (some of) the distances by neighborhood sequences on the square grid are sometimes referred as octagonal distances. Note that the size and the shape of the octagons do not depend on the order of steps.

\subsection{Known results}

In this subsection we recall some of the results about the number of shortest paths of those special cases which are described, e.g., by Das in [3].

Let $p$ and $q$ be two points such that their (absolute) coordinate differences are $i$ and $j$ in the first and in the second coordinates, respectively. Then, by cityblock distance, i.e., using the neighborhood sequence $B$ containing only 1 's, the distance is the sum of the coordinate differences, i.e., $i+j$. Further, the number of shortest paths is $\left(\begin{array}{c}i+j \\ i\end{array}\right)=\left(\begin{array}{c}i+j \\ j\end{array}\right)$, since the path contains $i$ vertical and $j$ horizontal moves, and their order is arbitrary. This result is also connected to shuffles of trajectories, see [8]. 
Das also counted the number of shortest path by the chessboard distance, and by the octagonal distance, recommended by Rosenfeld and Pfaltz, described by the neighborhood sequence $B=(1,2,1,2, \ldots)$ with $b(i)=1$ at each odd position ( $i=$ $2 k+1$ for $k \in \mathbb{N})$, and $b(i)=2$ at each even position $\left(i=2 k\right.$ with $\left.k \in \mathbb{N}^{+}\right)$. However, as it is written in [3] to count the the number of shortest paths in the general case left open.

We note that Das has obtained these results based on recursive formulation using recurrence relations and generating functions. Opposite to this, we will prove our formulae by enumerative combinatorial techniques. Moreover, we prove our result for more general cases, since Das and his co-authors used only periodic neighborhood sequences $([4,5]$, etc.), but the concept has also been generalized to not necessarily periodic neighborhood sequences (see e.g. [9]).

In the next sections we present our results.

\section{Algorithm to COMPUte THE NUMBER OF SHORTEST PATHS}

Since the $B$-distances are translation invariant (they depend only on the coordinate difference of the points and on the used neighborhood sequence), further we may assume that one of the points is the origin $(0,0)$.

We give an algorithm that also shows the recursive nature of the problem. Similarly to the way one can obtain digital disks (shown, e.g., in Fig. 1), instead of increasing the distance, one can count the number of shortest paths.

Actually, the hardness of the problem is hidden in the algorithm. At step 7 there are various cases how a point gets its value:

- If $b(i)=1$

- There are points for which there is exactly one of its 1-neighbors had assigned value already.

- There are points for which two of their 1-neighbors had already known value.

- If $b(i)=2$ there are three subcases:

- A point could have only one 2-neighbor with already known value.

- A point may have exactly two 2-neighbors with already assigned values.

- A point may have already three 2-neighbors with known values.

Fig. 2 shows one-one example for each of these cases. 


\section{Algorithm 1}

Input: a neighborhood sequence $B=(b(i))_{i=1}^{\infty}$ and a point $(x, y)$.

Output: the number of shortest paths from $(0,0)$ to $(x, y)$.

1 . Let the number of shortest paths to the origin $(0,0)$ be fixed as 1 .

2. Let $i=0$.

3. While the number of shortest paths is not assigned to $(x, y)$

Do

4. Let $i=i+1$.

5. Let $S$ be the set of points $p$ with both of the following properties:

5.a $\quad p$ does not have any value assigned yet, and

5.b $\quad p$ has at least one $b(i)$-neighbor with already assigned value.

6. For each element $p$ of $S$ do in parallel

7. Assign the sum of the known values of its $b(i)$-neighbors to $p$.

8. EndFor.

9. EndWhile.

As one may observe that with $b(i)=1$ at the straight line sides the value is copied (kept) from the previous element (Fig. 2, a), while at 'stairs' the sum of two previous values known at the neighbors are used (Fig. 2, b). In case $b(i)=2$, roughly saying, sum of 3 previous neighbor values are used for inner points of straight line sides (Fig. 2, c), while only one value is copied at the 'corners' (Fig. 2, e) and the sum of two values is used for neighbors of the corners (Fig. 2, d). At stair sides, however, the sum of three values of neighbors with previously assigned values is used at the inner points of the stairs and one value is copied for the outer points of the stairs (see Fig. $2, \mathrm{f}$ and $\mathrm{g}$, respectively).

We note here that the applied algorithm can be seen as a variation of Dijkstra algorithm and the breadth-first search algorithm, the correctness of the algorithm is also based on this fact. Actually, it sums up the number of shortest paths to the points from which the target point can be reached in one step in a shortest path (and it assigns the value, the number of shortest paths to these, previous points in a similar manner based on the number of shortest paths for those points from which they can be reached in by a last step in a shortest path, etc.) Also, the distance transform algorithms used in digital image processing are closely related. Fig. 2 highlights the complexity of the problem, comparing it, e.g., to the case of cityblock distances, where the binomial coefficients are obtained based on the Pascal triangle.

The time complexity of the algorithm is quadratic in the number of steps, i.e., the distance of the points. Actually, the value for each point can be computed within constant time (Steps 6 and 7): A point has a bounded number of $b(i)$-neighbors, i.e., at most 8 , but the number of those which has already value is always less than 8 . The sum of those values can be computed in a fixed amount of time (assuming a type that is large enough to work with these numbers). 


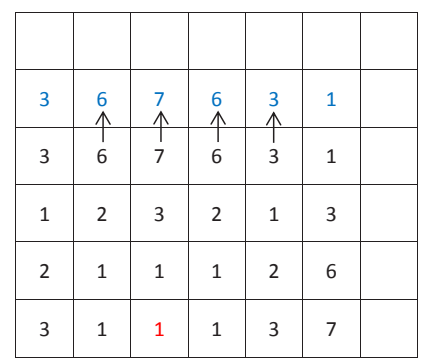

(a)

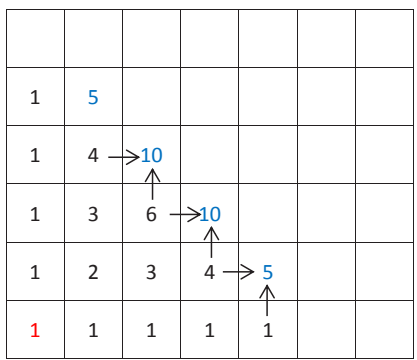

(b)

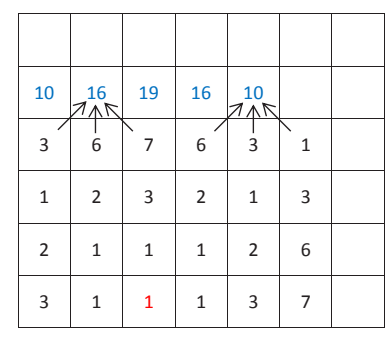

(c)

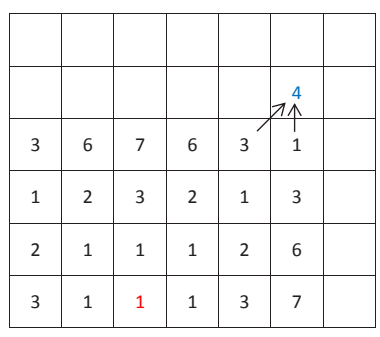

(d)

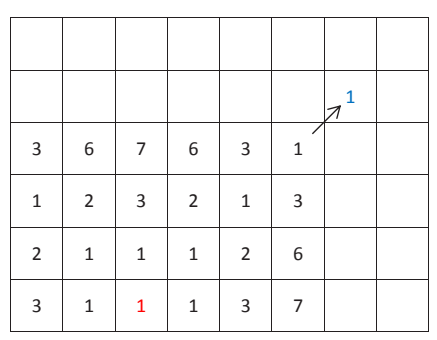

(e)

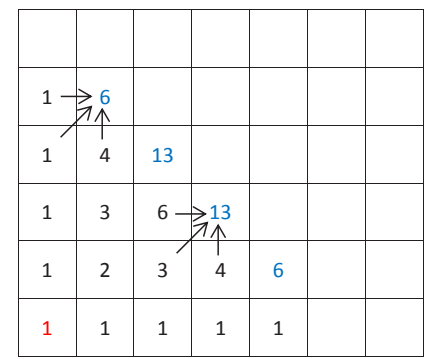

(f)

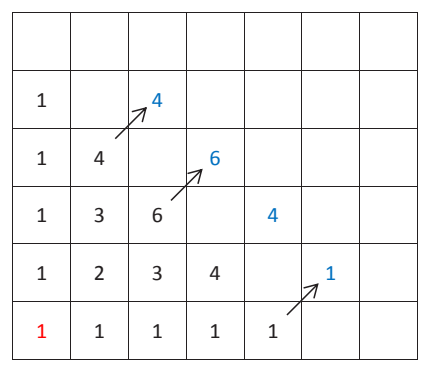

(g)

FIGURE 2. Examples for various cases from where the number of shortest paths can be summed up to a point (the origin is marked by red color in each figure, example new values are shown by blue). Cases with $b(i)=1$ are shown in (a) straight side, (b) stair side. Cases with $b(i)=2$ are shown in (c),(d),(e) straight side, (f), (g) stair side.

The number of points that have already assigned values is at most $(2 i+1)^{2}$, consequently the set $S$ has at most $4(2 i+1)=8 i+4$ elements. Depending on the used neighborhood sequence $B$, by reaching the point $(x, y)$, at $\operatorname{most}(2 \max \{|x|,|y|\}+1)^{2}$ points get their values. This shows the quadratic time and also the quadratic space complexity of the algorithm. 


\section{FORMULAE FOR THE NUMBER OF SHORTEST PATHS}

In this section we provide direct formulae to compute the number of shortest $B$ paths between any two gridpoints. The formula for the number of shortest paths can be computed in 3 different ways depending on the coordinate differences of the points and the number of 2's in the beginning part of the sequence $B$, i.e., in the part that is used to provide the shortest $B$-path(s).

The cases can be considered as follows.

Let the maximal coordinate difference of the two points ( $p$ and $q$ ) be $j$, and the other coordinate difference be $i$. Moreover, for simplicity, let $d=d_{B}(p, q)$ denote the $B$-distance of the points.

- If the number of 2's among the first $j$ elements of $B$ is exactly $i$, then this is the "fit case".

- We have the "poor case" if there are less 2's among the first $d$ elements of $B$ than $i$.

- In this case there are more 2's in the initial part of $B$ than the number of 2's that is needed in a shortest path. This is the "rich case".

Let us use the following notation. Let $k_{1}$ and $k_{2}$ denote the number of 1's and 2's among the first $k$ elements of $B$. Obviously $k_{1}+k_{2}=k$. (We will use this notation for $k=j$ and for $k=d$.)

Now, let us start with the simplest case.

\subsection{The fit case}

Proposition 1. Let $p, q \in \mathbb{Z}^{2}$ and $B$ be a neighborhood sequence. Denote $j=$ $\max \left\{\left|x_{p}-x_{q}\right|,\left|y_{p}-y_{q}\right|\right\}$ and $i=\min \left\{\left|x_{p}-x_{q}\right|,\left|y_{p}-y_{q}\right|\right\}$. If the number of 2 's among the first $j$ elements of $B$ is exactly $i$, then the B-distance of the points $p$ and $q$ is $d=j$.

This statement is a straightforward special case of Eq. 2.1. In a shortest $B$-path at each 1-step we move at the direction of the larger coordinate difference, and at each 2-step we have a diagonal movement decreasing both coordinate differences. Obviously, the conditions given in this case allow exactly the shortest paths with this property. Consequently, in this case on can establish the following result.

Theorem 1. Let $p, q \in \mathbb{Z}^{2}$ and $B$ be a neighborhood sequence. Denote $j=$ $\max \left\{\left|x_{p}-x_{q}\right|,\left|y_{p}-y_{q}\right|\right\}$ and $i=\min \left\{\left|x_{p}-x_{q}\right|,\left|y_{p}-y_{q}\right|\right\}$. If the number of 2 's among the first $j$ elements of $B$ is exactly $i$, then there is exactly 1 shortest $B$-path from $p$ to $q$.

Proof. By Proposition 1, the length of the shortest path(s) from $p$ to $q$ is exactly $j$. Among these $j$ steps there could be at most $i$ diagonal steps, and actually, it needs to be $i$ diagonal steps to reach the endpoint $q$ from $p$. Consequently, all steps decrease the coordinate difference in the direction of $j$ and all $i$ 2-steps decrease both 
coordinate differences. This can be done exactly in one way, as it is described, the direction of each step in the path is uniquely determined by $B$ in this case.

In this case, $d+d_{2}=i+j$ and also $d=j$, thus $i+j=j+j_{2}$ which leads to $i=j_{2}$.

Notice that there is exactly one $B$-path also from $q$ to $p$, however, this path not necessarily built up from the same points as the one from $p$ to $q$.

\subsection{The poor case}

If there are less 2's among the first $d$ elements of $B$ than $i$, then some of the "diagonal moves" (of the previous case) must be substituted by two 1-steps. Therefore, in this case, $d>j$.

We can rewrite formula Eq. 2.1 in the following form, for this case.

Proposition 2. Let $p, q \in \mathbb{Z}^{2}$ and $B$ be a neighborhood sequence. Denote $j=$ $\max \left\{\left|x_{p}-x_{q}\right|,\left|y_{p}-y_{q}\right|\right\}$ and $i=\min \left\{\left|x_{p}-x_{q}\right|,\left|y_{p}-y_{q}\right|\right\}$. If the number of 2 's among the first $j$ elements of $B$ is less than $i$, then the B-distance of the points $p$ and $q$ is $d=\min \left\{k \mid i+j \leq \sum_{n=1}^{k} b(n)\right\}$.

Roughly saying, there are not enough 2-steps for having enough diagonal steps for $i$, thus some 1's are needed for steps to that direction and not only for the direction of the coordinate difference $j$.

In this paper we are interested to count the number of shortest $B$-paths, and thus, it is important to recognise that there are two subcases:

- Matching case: The sum of the first $d$ elements of $B$ is exactly $i+j$ (with $\left.\sum_{n=1}^{d} b(n)=j+i\right)$.

- Mismatch case: The sum of the first $d$ elements of $B$ is larger than $i+j$. It can happen when the last used element of $B$, i.e., $b(d)$ is a 2 , but 1 would suffice. In this case, $\sum_{n=1}^{d} b(n)>j+i$, i.e., with more precision, $\sum_{n=1}^{d} b(n)=j+i+1$.

\subsubsection{Matching case}

Theorem 2. Let $p, q \in \mathbb{Z}^{2}$ and $B$ be a neighborhood sequence. Denote $j=$ $\max \left\{\left|x_{p}-x_{q}\right|,\left|y_{p}-y_{q}\right|\right\}$ and $i=\min \left\{\left|x_{p}-x_{q}\right|,\left|y_{p}-y_{q}\right|\right\}$ and let $d$ denote the $B-$ distance of $p$ and $q$. If the number of 2 's among the first $j$ elements of $B$ is less than $i$ and $\sum_{n=1}^{d} b(n)=j+i$, then the number of shortest paths from $p$ to $q$ is $\left(\begin{array}{c}d-d_{2} \\ i-d_{2}\end{array}\right)=\left(\begin{array}{c}d_{1} \\ i-d_{2}\end{array}\right)$.

Proof. The length of a shortest path is $d$, and, since $\sum_{n=1}^{d} b(n)=j+i$, every 2-steps must be a diagonal step by decreasing both the coordinate differences. All 1-steps, their number is $d_{1}=d-d_{2}$, must also decrease one of the coordinate differences. 
Actually, in the shortest paths $i-d_{2}$ is the number of 1-steps to the direction of the coordinate difference $i$ (and the remaining 1-steps are used in the direction determined by $j$ ). The order of these 1-steps is arbitrary, only their number is important to reach $q$ from $p: i-d_{2}$ steps corresponding to direction $i$ and $d_{1}-\left(i-d_{2}\right)=d-i$ steps to the orthogonal direction, from the $d_{1} 1$-steps, that is, $\frac{d_{1} !}{\left(i-d_{2}\right) !(d-i) !}=\left(\begin{array}{c}d_{1} \\ i-d_{2}\end{array}\right)$. Thus the number of shortest path can be written by the binomial coefficients given in the theorem.

Notice that in this case $i+j=2 d_{2}+d_{1}$ holds, which, combined by $d=d_{2}+d_{1}$ and knowing $j<d$, gives $d=j+i-d_{2}$ with $i-d_{2}>0$.

\subsubsection{Mismatch case}

In this case the sum of the first $d$ elements of $B$ is $i+j+1$.

Theorem 3. Let $p, q, B$ be given and the notation $j, i, d$ be the same as at Theorem 2. If the number of 2's among the first $j$ elements of $B$ is less than $i$ and $\sum_{n=1}^{d} b(n)>$ $j+i$, then the number of shortest paths from $p$ to $q$ is $d_{2}\left(\begin{array}{c}d+1-d_{2} \\ i+1-d_{2}\end{array}\right)$.

Proof. The length of a shortest path is $d$, but $\sum_{n=1}^{d} b(n)>j+i$, thus, there is exactly one 2-step which is actually, a 1-step. All other 2-steps must be a diagonal step by decreasing both the coordinate differences. The number of choices to find which 2step is a 1-step in the path, i.e., to replace a value 2 to 1 among the first $d$ elements of $B$, is exactly $d_{2}$. After, the replacement of this one element, actually, the number of shortest $B$-paths can be computed by Theorem 2, since the sum of $i+j$ matches to the modified neighborhood sequence. Consequently, $\left(\begin{array}{c}d+1-d_{2} \\ i+1-d_{2}\end{array}\right)$ gives the number of shortest paths for a modified neighborhood sequence: $d+1-d_{2}$ is the number of 1steps in the shortest paths and $i+1-d_{2}$ is the number of 1-steps to the direction of the coordinate difference $i$. Using the multiplication rule for choosing first one element 2 , and then the directions of the 1-steps, the formula of the theorem follows.

\subsection{The rich case}

This is the most complex case, although the distance is exactly $d=j$ since there are enough 2's for the diagonal steps. However, there are extra value(s) 2 in the sequence. In shortest paths each 1-step is in the direction of the coordinate difference of $j$ (otherwise $j$ steps would not be enough). However, the 2 -steps gives more variety for the shortest paths.

Theorem 4. Let $p, q \in \mathbb{Z}^{2}$ and $B$ be a neighborhood sequence. Denote $j=\max \left\{\left|x_{p}-x_{q}\right|,\left|y_{p}-y_{q}\right|\right\}$ and $i=\min \left\{\left|x_{p}-x_{q}\right|,\left|y_{p}-y_{q}\right|\right\}$. If the number of 2 's among the first $j$ elements of $B$, i.e., $j_{2}$, is more than $i$, then the number of shortest 
B-paths from $p$ to $q$ can be computed as

$$
\sum_{m=i}^{i+\left\lfloor\frac{j_{2}-i}{2}\right\rfloor} \frac{j_{2} !}{m !(m-i) !\left(j_{2}+i-2 m\right) !} .
$$

Proof. Let us start with a shortest path that can be obtained by the greedy algorithm $([9,10])$. In this $B$-path, the first $i$ 2-steps are diagonal steps decreasing the coordinate difference in both coordinates (we call the 2-steps of this direction as 'good' 2-steps), and all other steps are 1-steps decreasing also the coordinate difference on the direction determined by $j$.

But now, we are looking for the number of the shortest paths without caring about if they could be generated by the greedy algorithm or not. So, definitely, the 1-steps in any shortest path must be in the direction given by $j$. For the 2 -steps there are three possibilities:

- it is a 'good' 2-step: a diagonal step according the difference in both directions;

- it is a 1-step to the direction of $j$;

- it is a 'bad' 2-step, i.e., it is a diagonal step in the direction according to $j$, but opposite to the direction determined by $i$.

There cannot be other types of 2-steps which decrease the coordinate difference of $j$, and thus providing shortest paths.

Let $m$ be the number of 'good' 2-steps in a shortest $B$-path, then there must be $n$ 'bad' 2-steps also in the path to reach exactly $q$ from $p$ in $j$ steps, such that $m-n=i$. Then, $n=m-i$. The condition (the number of diagonal steps in the path) $m+n \leq j_{2}$ must also be fulfilled. In such a path there are $j_{2}-m-n=j_{2}+i-2 m$ 2-steps that are actually 1 -steps.

The value of $m$ must be at least $i$ (both $m, n$ are nonnegative) and further, at most $\left\lfloor\frac{j_{2}-i}{2}\right\rfloor$ pairs of 'good' and 'bad' 2 -steps could be added as extra diagonal steps. The order of these 2-steps can be arbitrary (among the 2-steps allowed by $B$ ). Consequently, by fixing the value $m$ of 'good' 2 -steps, the formula $\frac{j j_{2} !}{m !(m-i) !\left(j_{2}+i-2 m\right) !}$ gives the number of shortest paths with that number of that type of steps. Now, by summing up the values for the possible values of $m$, the formula is obtained that we wanted to prove.

Observe that the fit case can also be seen as a special case of this, rich case, as the formula of Theorem 4 gives 1 in the case $j_{2}=i$.

Also the case of chessboard distance (investigated already by Das) is a special case of the rich case with $j_{2}=j$ : Thus, using the notation of Theorem 4 , the number of 
shortest paths from $p$ to $q$ by the chessboard metric is given by

$$
\sum_{m=i}^{i+\left\lfloor\frac{j-i}{2}\right\rfloor} \frac{j !}{m !(m-i) !(j+i-2 m) !},
$$

which, as one can easily check, coincides with Das's formula [3]. Das also used the abbreviation with "trinomial coefficients", due to the fact that instead of the sum of two values (as at the usual binomial coefficients), the sum of three earlier values are used. The trinomial coefficients are defined as $\left(\begin{array}{c}i+j+k \\ i, j, k\end{array}\right)=\frac{(i+j+k) !}{i ! j ! k !}$ and the formula for counting the chessboard distances can be written in a somewhat shorter form:

$$
\sum_{m=i}^{i+\left\lfloor\frac{j-i}{2}\right\rfloor}\left(\begin{array}{c}
j \\
m, m-i, j+i-2 m
\end{array}\right) .
$$

\subsection{Examples}

We close this section by some examples. Let $B=(1,1,2,1,2,1,2,2,2,2,2,1,1,1,2, \ldots)$ and $p$ be the origin.

Let $q_{1}=(7,3)$. Clearly, it is the fit case, since there are three 2's among the first seven elements of $B$. Thus, there is exactly one shortest $B$-path, and it is $((0,0),(1,0),(2,0),(3,1),(4,1),(5,2),(6,2),(7,3))$.

Let $q_{2}=(11,12)$. We are in the poor case, $d=15$ and it is the matching subcase (the sum of the first fifteen elements is exactly 23). We have $i=11$ and $d_{2}=8$. Thus, by Theorem 2, the number of shortest $B$-paths is $\left(\begin{array}{c}7 \\ 11-8\end{array}\right)=\left(\begin{array}{l}7 \\ 3\end{array}\right)=35$.

Now, let $q_{3}=(6,3)$, and thus, $j=6, i=3$. This is the poor case, since there are only two 2's among the first six elements. One may check that $d=7$, and thus we have the mismatch case, the sum of the first seven elements is 10 , larger than $j+i=6+3=9$. Also $d_{2}=3$. Thus, by Theorem $3,3\left(\begin{array}{c}7+1-3 \\ 3+1-3\end{array}\right)=5$.

Finally, let $q_{4}=(10,4)$. Now, it can be computed according to the rich case (there are six 2's among the first ten elements of $B$ ). By our notation $j_{2}=6$ and $i=4$. Thus, by Theorem 4, we have $\sum_{m=4}^{5} \frac{6 !}{m !(m-4) !(10-2 m) !}=\frac{6 !}{4 ! 0 ! 2 !}+\frac{6 !}{5 ! 1 ! 0 !}=\frac{6 \cdot 5}{2}+6=21$.

In Fig. 3 we show some values for the shortest paths with chessboard distance. Colors show the pixels that can be reached by various number of steps, e.g., red for one step, dark blue for two steps, green for three steps, etc. from the shaded pixel.

\section{CONCLUSiOnS}

A combinatorial problem was considered (coined originally by Das in 1991 as an open problem for periodic neighborhood sequences) and solved (also for not necessarily periodic neighborhood sequences): Enumerative combinatorics is used to compute the number of shortest paths for digital distance functions defined by neighborhood sequences. 


\begin{tabular}{|c|c|c|c|c|c|c|c|c|c|c|c|c|c|}
\hline 112 & 266 & 504 & 784 & 1016 & 1107 & 1016 & 784 & 504 & 266 & 112 & 36 & 8 & 1 \\
\hline 28 & 77 & 161 & 266 & 357 & 393 & 357 & 266 & 161 & 77 & 28 & 7 & 1 & 8 \\
\hline 6 & 21 & 50 & 90 & 126 & 141 & 126 & 90 & 50 & 21 & 6 & 1 & 7 & 36 \\
\hline 1 & 5 & 15 & 30 & 45 & 51 & 45 & 30 & 15 & 5 & 1 & 6 & 28 & 112 \\
\hline 5 & 1 & 4 & 10 & 16 & 19 & 16 & 10 & 4 & 1 & 5 & 21 & 77 & 266 \\
\hline 15 & 4 & 1 & 3 & 6 & 7 & 6 & 3 & 1 & 4 & 15 & 50 & 161 & 504 \\
\hline 30 & 10 & 3 & 1 & 2 & 3 & 2 & 1 & 3 & 10 & 30 & 90 & 266 & 784 \\
\hline 45 & 16 & 6 & 2 & 1 & 1 & 1 & 2 & 6 & 16 & 45 & 126 & 357 & 1016 \\
\hline 51 & 19 & 7 & 3 & 1 & 1 & 1 & 3 & 7 & 19 & 51 & 141 & 393 & 1107 \\
\hline 45 & 16 & 6 & 2 & 1 & 1 & 1 & 2 & 6 & 16 & 45 & 126 & 357 & 1016 \\
\hline 30 & 10 & 3 & 1 & 2 & 3 & 2 & 1 & 3 & 10 & 30 & 90 & 266 & 784 \\
\hline 15 & 4 & 1 & 3 & 6 & 7 & 6 & 3 & 1 & 4 & 15 & 50 & 161 & 504 \\
\hline
\end{tabular}

FIGURE 3. A segment of the square grid by the number of shortest chessboard paths from the shaded pixel. Our Algorithm can be applied to fill the grid, layer by layer in a parallel manner (as the colors indicate).

When the number of 2's in the prefix of the neighborhood sequence $B$ is less than the smaller (absolute) coordinate difference, i.e., in the poor case, the number of minimal paths, can be considered as the combination based on the 1-steps: among the 1-steps some of them change the first coordinate, the others change the second coordinate, and the order of these steps can be arbitrary (among the 1-steps). Consequently, the formula can be written by binomial coefficients and closely related to the case of cityblock distances.

With sufficiently large number of 2's in the prefix of $B$, the formula gives summations on the possible numbers of diagonal-steps and 1-steps made with values 2 of $B$. These results are more related to the case of chessboard distance. As a special case of this, in the fit case (when the smaller coordinate difference gives exactly the number of 2's in the prefix of $B$ of the length that is exactly the larger coordinate difference) there is exactly 1 shortest $B$-path.

The results can be seen as generalisations of binomial (and trinomial) coefficients based on the used distance functions. 
We should also note that counting the number of shortest path is not only a combinatorial exercise, but also has strong image processing applications (see, e.g., [16]). We mention that in [1] a similar problem was studied for other popular digital distances, namely for the chamfer distances. Furthermore, counting the shortest paths has also been started in other grids, see e.g., [6] for the first results on the triangular grid.

\section{ACKNOWLEDGEMENTS}

The main results of this paper were presented in the 1st Joint Austrian-Hungarian Mathematical Conference (AUSHUN'15), Győr, Hungary in August, 2015. Comments of the audience and advice of the anonymous reviewer are gratefully acknowledged.

\section{REFERENCES}

[1] L. Alzboon, B. Khassawneh, and B. Nagy, "On the number of weighted shortest paths in the square grid," in 2017 IEEE 21st International Conference on Intelligent Engineering Systems (INES)," doi: 10.1109/INES.2017.8118533, 2017, pp. 83-90.

[2] P. Das, "An algorithm for computing the number of the minimal paths in digital images." Pat. Recog. Let., vol. 9, pp. 107-116, 1989.

[3] P. Das, "Counting minimal paths in digital geometry." Pat. Recog. Let., vol. 12, pp. 595-603, 1991.

[4] P. Das, P. Chakrabarti, and B. Chatterji, "Distance functions in digital geometry." Inform. Sci., vol. 42, pp. 113-136, 1987.

[5] P. Das, P. Chakrabarti, and B. Chatterji, "Generalized distances in digital geometry." Inform. Sci., vol. 42, pp. 51-67, 1987.

[6] M. Dutt, A. Biswas, and B. Nagy, "Number of shortest paths in triangular grid for 1- and 2neighborhoods," IWCIA 2015, LNCS, vol. 9448, pp. 115-124, 2015, doi: 10.1007/978-3-31926145-4_9.

[7] A. Hajdu, B. Nagy, and Z. Zörgő, "Indexing and segmenting colour images using neighbourhood sequences." ICIP'03, vol. I, pp. 957-960, 2003.

[8] A. Mateescu, G. Rozenberg, and A. Salomaa, "Shuffle on trajectories: Syntactic constraints." Theor. Comp. Sci., vol. 197, pp. 1-56, 1998.

[9] B. Nagy, "Distance functions based on generalized neighbourhood sequences in finite and infinite dimensional space." ICAI'01, vol. Eger, pp. 183-190, 2001.

[10] B. Nagy, "Distance functions based on neighbourhood sequences." Publ. Math. Debrecen, vol. 63, no. 3, pp. 483-493, 2003.

[11] B. Nagy, "Metric and non-metric distances on $\mathbb{Z}^{n}$ by generalized neighbourhood sequences." ISPA 2005, vol. IV, pp. 215-220, 2005.

[12] B. Nagy, "Distances with generalized neighbourhood sequences in $n \mathrm{D}$ and $\infty \mathrm{D}$." Disc. Appl. Math., vol. 156, pp. 2344-2351, 2008, doi: 10.1016/j.dam.2007.10.017.

[13] B. Nagy, "Application of neighborhood sequences in communication of hexagonal networks," Disc. Appl. Math., vol. 216, pp. 424-440, 2017, doi: 10.1016/j.dam.2015.10.034.

[14] B. Nagy, "Generalized distances of sequences I: B-distances." Miskolc Math. Notes, vol. 19, pp. 397-411, 2018.

[15] B. Nagy, "Generalized distances of sequences II: B-distances with weight sequences." Filomat, vol. 33, no. 18, pp. 5803-5812, 2019. 
[16] A. Rosenfeld and J. Pfaltz, "Distance functions on digital pictures." Pat. Recog., vol. 1, pp. 33-61, 1968.

[17] R. Strand, B. Nagy, C. Fouard, and G. Borgefors, "Generating distance maps with neighbourhood sequences," DGCI 2006, LNCS, vol. 4245, pp. 295-307, 2006, doi: 10.1007/11907350_25.

[18] M. Yamashita and T. Ibaraki, "Distances defined by neighborhood sequences." Pat. Recog., vol. 19, no. 3, pp. 237-246, 1986.

[19] M. Yamashita and N. Honda, "Distance functions defined by variable neighborhood sequences," Pat. Recog., vol. 17, no. 5, pp. 509-513, 1984, doi: 10.1016/0031-3203(84)90048-7.

Author's address

Benedek Nagy

Eastern Mediterranean University, Faculty of Arts and Sciences, Department of Mathematics, Famagusta, North Cyprus, via Mersin-10, Turkey

E-mail address: nbenedek.inf@gmail.com 